\title{
CASE OF CHOLECYSTOTOMY
}

PERFORMED FOR

\section{DROPSY OF THE GALL-BLADDER DUE TO IMPACTION OF A GALL-STONE.}

BP

LAWSON TAIT, F.R.C.S., \&c., SURGEON TO THB BIRMINGHAM HOSPITAL FOR WOMRN.

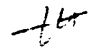

(Receired October 14th-Read November 11th, 1879.) $f$

THe great success which has followed the performance of operations for ovarian tumours has led to the extended use of abdominal section in directions which could hardly have been hoped for till within the last few years. My own experience, of now nearly two hundred abdominal sections, has been such as to lead me to this conclusion, that no abdominal or pelvic tumour ought to be left unexplored, if it seems to affect seriously the life and comfort of the patient, unless it be incontestibly of a cancerous character.

Dr. Marion Sims attributes to Dr. Handfield Jones, and I think correctly, the merit of first suggesting that the liver and gall-bladder should be included within the field of surgical practice more fully than they had been up to that point, and particularly that surgical interference should be made in cases where death is threatened from the impaction of a gall-stone.

To Dr. Marion Sims himself must be given the credit of having followed out this suggestion with his usual VOL. LXIII. 
boldness and ability, and he himself points out that the case in which he did it was not successful, only because the operation was too long delayed.

To my good fortune it has fallen to be the first to follow out Dr. Handfield Jones' idea and Dr. Sims' plan successfully.

Elizabeth M-, æt. 40, was admitted to the hospital on Angust 18th, having been sent to me by Dr. Abraham Colles, of Bridgnorth, on account of an abdominal tumour.

She had been married eighteen years, had borne six children, her menstruation had always been normal, and she had enjoyed perfectly good health until the summer of 1878. At that time she began to suffer from severe spasmodic pains in the right side, these being always aggravated by walking or by lifting even slight weights. In September she noticed a swelling at the seat of pain, and this slowly increased. During last winter her pain became much more intense, her appetite failed, she lost strength and flesh rapidly, and on admission she presented an emaciated and almost cachectic appearance. She also suffered at that time from incessant headache and sickness, and obstinate constipation. The seat of pain was over the right kidney, where there was a heart-shaped tumour, firm and elastic, in which no fluctuation could be detected, and which was extremely tender to the touch. On examination under ether, this tumour was found to be perfectly movable towards each side, indeed, it could be pushed completely across the middle line to the left side. All round it a note of intestinal resonance could be produced. When pushed over to the left side, its heartlike shape became very apparent, and when it lay on the left side of the vertebral column, with its apex directed downwards and to the left, its base evidently retained a connection with the right side.

A careful examination of the urine gave only negative results, though she spoke vaguely of its having been occasionally dark in colour, muddy, and deficient in quantity. 
At the consultation, held with my colleague Dr. Edginton, upon the case, a variety of suggestions were made for diagnosis, the chief of which were cystic enlargement of a floating kidney, a tumour of the head of the pancreas, and dropsy of the gall-bladder. But no decided diagnosis was attempted, and my proposal to open the abdomen, and thus ascertain the nature of the tumour, was agreed upon.

On August 23rd I opened the abdomen in the middle line, to the extent of four inches, the umbilicus forming the centre of the incision. It then became at once evident that the tumour was a distended gall-bladder. I passed the needle of an aspirator into the apex and drew off a quantity of white starchy-looking fluid, probably amounting to between twelve and fifteen ounces, but I cannot speak positively as to its amount, as it was unfortunately thrown away by a nurse immediately after the operation. I then opened the gall-bladder at the point of puncture, so as to admit my finger, and came at once upon a large round gall-stone lying loose in the cavity. This I easily removed, and on further search I found another of rather larger size, and probably of pear-shape, at the entrance of the duct, impacted in it, and evidently the cause of the dropsical distension of the gall-bladder. The removal of this stone was a matter of very great difficulty, in fact, it took a very much longer time to effect than all the other steps of the operation put together. From the long, narrow, funnel-like cavity in which it was lodged, and from the mobility of the bladder, it was very difficult to seize, and when at last I did got hold of it I found it adherent to the mucous surface. I had then to consider the extreme likelihood that in removing this impacted stone I might tear the walls to which it was attached, and thus certainly kill my patient. I therefore performed a very careful and protracted lithotrity, chipping little fragments off the stone regularly all over its exposed surface till I had the satisfaction of lifting out its nucleus. I then passed a blade of a fine 
pair of forceps on each side of it, and by a gentle squeeze broke up the remainder, and was then enabled to lift it all out. The weight of the stone removed entire is 4.2 grammes, and that of the fragments I could gather of the broken stone is 2.9 grammes, but of the latter stone as much again must have been lust on the sponges which were packed into the wound during the process of crushing, and upon. which I had constantly to wipe my instruments. I washed the cavity out repeatedly and took every precaution that I could to secure that no fragments were left. I then stitched the wound in the gall-bladder to the upper end of the wound in the abdominal walls by continuous sutures, leaving the aperture into the bladder quite open, and then I closed the rest of the abdominal opening in the usual way. The operation was performed with complete antiseptic precautions, and the anæsthetic employed was other.

She rallied from the operation completely in a few hours. I dressed the wound antiseptically the same evening at 11 p.m., and found the dressings stained with healthy bile. In the further progress of the case there is very little to report, save that the flow of bile from the wound continued till the 3rd of September, when the dressings were discontinued and zinc ointment was used in their place. The stitches were removed and the wound was completely healed on September 9 th, when she began to take solid food, up to that time her diet having been restricted to milk and beef tea. On the 14th she sat up for the first time, and on the 30th she went home quite restored to health, free from pain and all her former symptoms, and having gained at least a stone in weight.

The annexed temperature chart is the best indication of the evenness and rapidity of her recovery.

Looking back upon this case, I do not think that a more accurate diagnosis was possible, for there was an entire absence of those symptoms which usually characterise cases of gall-stone. After the operation she told us that one of her neighbours had said to her one 


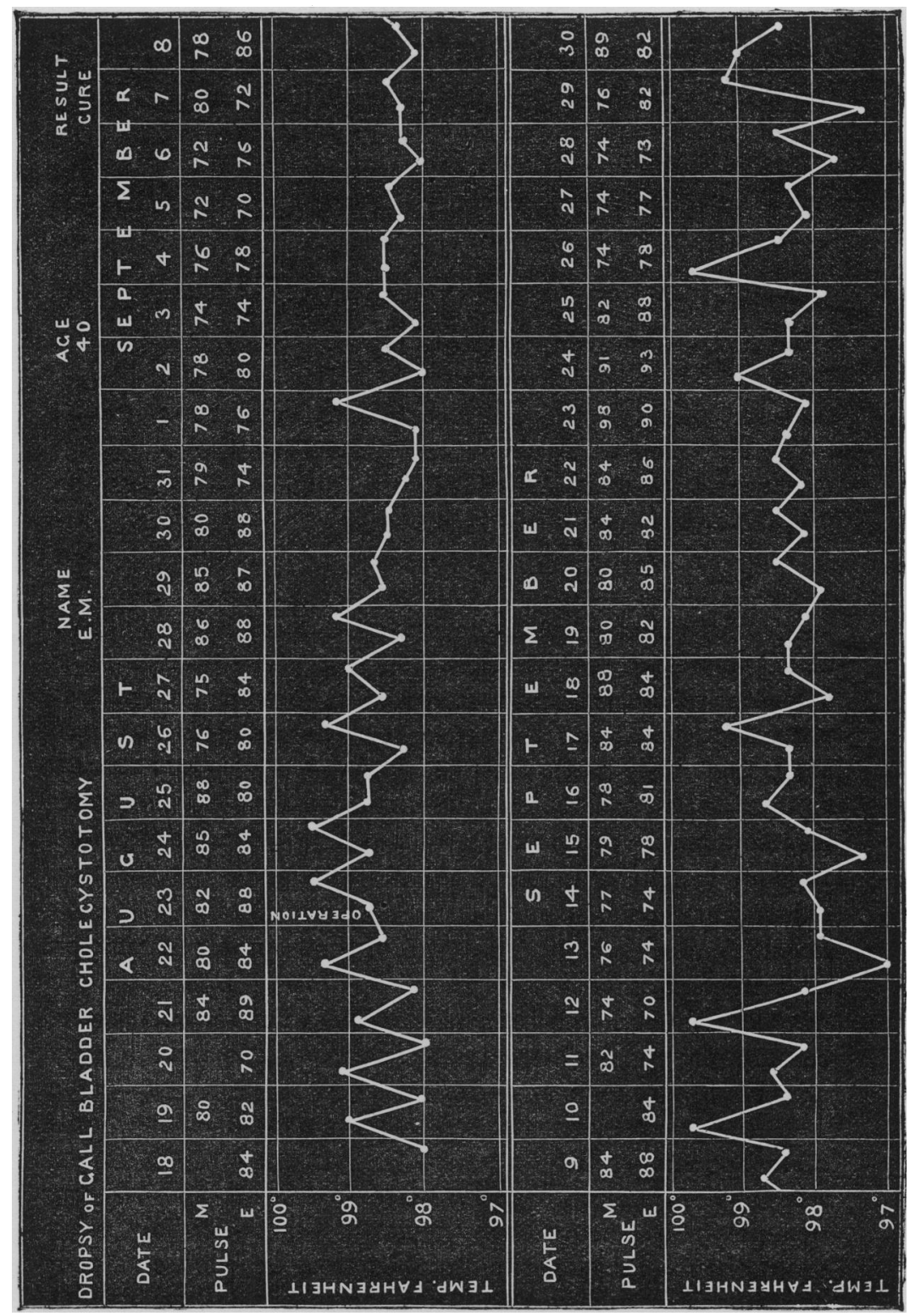


day that she thought the patient was jaundiced, but beyond this no history could be got at of any symptoms pointing clearly to the true nature of the case. The singular mobility of the tumour was also a most puzzling condition. Fortunately, our advanced practice in abdominal surgery makes our limited powers of diagnosis in such a case of less importance, and I thoroughly agree with Dr. Sims that we should not wait till the approach of almost fatal symptoms puts the diagnosis in unmistakable fashion, but that "we shall make an early exploratory incision, ascertain the true nature of the disease, and then carry out the surgical treatment that the necessities of the case may demand."

I have only one other point to speak of, and that is that the operation was done with rigid antiseptic precautions, as all my abdominal sections have for some time been. It would be foreign to my purpose here to enter into a discussion for which I do not think my own experience is as yet ripe, but the impression is growing upon me that my increasing success in these operations has little or nothing to do with the antiseptic system. I use it, however, with the utmost rigour, in order that my patients may have every chance for their lives, and because I have as yet had insufficient evidence that it does them any harm beyond delaying the healing of wounds, as it almost invariably does. 